\title{
Gradient twinned 304 stainless steels for high strength and high
}

\section{ductility}

Aiying Chen ${ }^{1}$, Jiabin $\mathrm{Liu}^{2}$, Hongtao Wang ${ }^{2}$, Jian $\mathrm{Lu}^{3 *}$, Y. Morris Wang ${ }^{4 *}$

${ }^{1}$ School of Materials Science and Engineering, University of Shanghai for Science and

Technology, Shanghai, China

${ }^{2}$ Institute of Applied Mechanics, Zhejiang University, Hangzhou, China

${ }^{3}$ Department of Mechanical and Biomedical Engineering, City University of Hong Kong, Hong Kong, China

${ }^{4}$ Physical and Life Sciences Directorate, Lawrence Livermore National Laboratory, Livermore, California, USA

\begin{abstract}
Gradient materials often have attractive mechanical properties that outperform uniform microstructure counterparts. It remains a difficult task to investigate and compare the performance of various gradient microstructures due to the difficulty of fabrication, the wide range of length scales involved, and their respective volume percentage variations. We have investigated four types of gradient microstructures in 304 stainless steels that utilize submicrotwins, nanotwins, nanocrystals, ultrafine- and coarse-grains as building blocks. Tensile tests reveal that the gradient microstructure consisting of submicrotwins and nanotwins has a persistent and stable work hardening rate and yields an impressive combination of high strength and high ductility, leading to a toughness that is nearly $50 \%$ higher than the coarse-grained counterpart. Ex- and in-situ transmission electron microscopy indicates that nanoscale and submicroscale twins help to suppress and limit martensitic phase transformation via the confinement of martensite within the twin lamellar. Twinning and detwinning remain active during tensile deformation and contribute to the work hardening behavior. We discuss the advantageous properties of using submicrotwins as the main load carrier and nanotwins as the strengthening layers over those coarse and nanocrystalline grains. Our work uncovers a new gradient design strategy to help metals and alloys achieve high strength and high ductility.
\end{abstract}

Key words: gradient materials; nano- and submicro-twins; in-situ TEM; high strength and high ductility; martensitic phase transformation

*Email: jianlu@cityu.edu.hk, ymwang@1lnl.gov 


\section{Introduction}

Materials with gradient or hierarchical microstructure often have far super mechanical properties than those of monolithic nanostructured materials, and have attracted rising interest in materials research community [1-3]. Such gradient microstructure can be formed thorough different grain sizes [4, 5], diverse twin spacing [6], or a combination of micro- and nano-sized grains and twins [6]. It has been conjectured and experimentally demonstrated that gradient hierarchical microstructure has the potential to evade the strength-ductility tradeoff dilemma in materials science $[3,5,6]$. Unlikely conventional materials, however, it is nontrivial to fabricate highly controlled gradient and more sophisticated hierarchical microstructures [3]. A systematic study of various gradient structures is obviously desirable but remains to be a daunting task due to a vast combination of different length scales and their volume fractions. As such, there exists little experimental effort to tackle this challenging problem.

Nanotwinned (NT) metals (defined as materials with average twin spacing $\lambda<100 \mathrm{~nm}$ ) are strengthened primarily by coherent twin boundaries (CTBs) and also known for their combination of good strength and ductility, high electrical conductivity, and high thermal stability [7]. NT-metals can be considered inherently as materials with multiple/hierarchical length scales [8], as the grain space parallel and vertical to CTBs is vastly different. This trait of NT-metals offers ample room for strain hardening and thus high ductility. One major limitation of NT-metals is that high-density twins are predominantly formed in medium- to low-stacking- fault energy materials [9]. For this reason, bulk NT structures have only been realized in a handful of metals, limiting their broad applications. For practical utility purpose, a bulk part of studies on NT-metals have been focused on model pure metals such as copper and silver $[7,10]$, which may bear less engineering applications than alloys such as steels. Questions remain as to how to take advantage of CTBs to achieve extraordinary mechanical properties in many real-life structural materials. In light of the emerging research on gradient microstructure and 
given the fact that CTBs exist in many metals and alloys (not necessarily with high density and in bulk form), an investigation of gradient hierarchical microstructures that contain CTBs is expected to generate novel mechanical properties and yield scientific insights for technological applications.

In this work, we systematically investigate the gradient hierarchical structures in 304 stainless steel (304 SS) (also known as A2 stainless steel), a material that is widely used by industry and home such as machinery parts and screws. 304 SS is a super corrosion-resistant material but relatively soft in coarse-grained (CG) form with a yield strength as low as $\sim 270 \mathrm{MPa}[11]$. Enhancing its strength has been the focus of many past studies (see refs.[12-20]). Unfortunately, strength and toughness are generally mutually exclusive [21]. The key is to increase the strength of these materials without sacrificing their useful tensile ductility (i.e., high toughness). Novel microstructure design strategies are much needed in order to retain both high strength and high toughness of 304 SS or other steels at large. Although gradient microstructure has been shown to have good strength, good ductility, and better fatigue life [5, 6, 22], it is unclear what type of gradient structures offer better solutions to above problems. For the first time, we report that a gradient microstructure comprises of NTs $(\lambda<100 \mathrm{~nm})$ and submicrotwins (STs, $100 \mathrm{~nm}<\lambda<1000 \mathrm{~nm}$ ) exhibit impressively higher strength and toughness than other gradient microstructures that contain nanocrystalline (NC), ultrafine-grained (UFG), and CG structures. We investigate the mechanistic insights of these observations using $e x-$ and in-situ transmission electron microscopy (TEM) and discuss their implications to other materials in general.

\section{Materials and methods}

\subsection{Four types of hierarchical microstructures}

As illustrated schematically in Fig. 1, we have investigated four types of 304 SS with multiple length scales, from ST (Type-I) microstructure to the one (Type-IV) that involves four different length scales, including NC (grain size $d<100 \mathrm{~nm}$ ), UFG (100 
$\mathrm{nm}<d<1000 \mathrm{~nm}), \mathrm{ST}$ and CG $(\mathrm{d}>1 \mu \mathrm{m})$ structures. Note that akin to NT structure, ST itself also contains multiple length scales, including a broad distribution of $\lambda$. For description simplicity, however, here we refer to Type-I as the material with one featured microstructure (i.e., ST); Type-II has two featured length scales (i.e., ST and NT), and Type-III and Type-IV have three and four featured length scales, respectively. Our baseline material is the ST 304 SS (Type-I). While metals strengthened by NTs have been broadly investigated, the studies of ST-strengthened microstructure are less often but could bear broader implications to engineering applications. Our Type-II material mixes ST with NT and forms one of the simplest gradient microstructure. In Type-III 304 $\mathrm{SS}$ (i.e., $\mathrm{ST}+\mathrm{NT}+\mathrm{NC}$ ), we investigate whether NC layers can further enhance the strength and toughness. Finally, a rather complex microstructure is studied; i.e., Type-IV $(\mathrm{ST}+\mathrm{NC}+\mathrm{UFG}+\mathrm{CG})$ with length scale spanning approximately three orders of magnitude from tens of microns to nanometers. In this design, ST and CG are expected to serve as plastic layers, whereas NC grains act as the main strengthening agent and UFG as a glue layer.

\subsection{Materials fabrication}

The chemical composition of $304 \mathrm{SS}$ is $0.04 \mathrm{C}, 0.49 \mathrm{Si}, 1.65 \mathrm{Mn}, 7.8 \mathrm{Ni}, 16.8 \mathrm{Cr}$, 0.37 Mo and balanced Fe (all in wt.\%). The 304 SS sheets with dimensions of $70 \mathrm{~mm} \times$ $50 \mathrm{~mm} \times 1 \mathrm{~mm}$ and $100 \mathrm{~mm} \times 90 \mathrm{~mm} \times 1 \mathrm{~mm}$ were bombarded via surface mechanical attrition treatment (SMAT) to prepare type-III and IV steels, respectively. Different ball type, diameter, and vibrating frequency are used. The detailed processing parameters of SMAT has been reported in ref. [11]. Both sides of the 304 SS sheets were treated. The Type-I and II steels were obtained by removing $270 \mu \mathrm{m}$ and $150 \mu \mathrm{m}$ thickness from both surfaces of Type-III SMAT steels, respectively (see Fig. 1).

\subsection{Tensile property measurements}

Tensile samples of Type-III and IV steels were cut into dogbone shape with a gauge length of $30.0 \mathrm{~mm}$ and a width of $5.0 \mathrm{~mm}$. For Type-I and Type-II $304 \mathrm{SS}$, the dogbone 
specimens were machined from Type-III steels with a gauge length of $6 \mathrm{~mm}$, and the surfaces were polished to remove NC and/or NT layers. The dimensions of tensile samples for Type-I and II 304 SS are $6.0 \mathrm{~mm} \times 1.0 \mathrm{~mm} \times 0.4 \mathrm{~mm}$ and $6.0 \mathrm{~mm} \times 1.0 \mathrm{~mm} \times 0.7$ mm, respectively. All tensile tests were performed using an MTS Alliance RT/50 testing system at a strain rate of $6.7 \times 10^{-4} \mathrm{~s}^{-1}$ and at room temperature. At least three tests were completed for each specimen type.

\subsection{Microstructure characterization}

In order to review the overall ST and NT structures, the cross-section of Type-II specimen was etched in solution $\left(2 \mathrm{ml} \mathrm{HF}+3 \mathrm{ml} \mathrm{HNO}_{3}+95 \mathrm{ml} \mathrm{H}_{2} \mathrm{O}\right)$ and examined via scanning electron microscopy (SEM, Hitachi S-4200). Conventional TEM was carried out using a JOEL-2010 microscope with an operating voltage of $200 \mathrm{kV}$. The $\lambda$ was measured from TEM images when $\lambda<1 \mu \mathrm{m}$, and by SEM when $\lambda$ in the micrometer range. As not all grains contain CTBs, the twin density (the percentage of grains containing twins) is an important parameter. We estimated the twin density at different depths by calculating the area fraction of grains containing twin bundles $(\lambda<1 \mu \mathrm{m})$. In-situ TEM tensile tests were performed in a JOEL-2100 TEM operated at $200 \mathrm{kV}$ using a Gatan 654 straining holder. The strain during the in-situ experiments is manually controlled at $0.5 \%$ strain every $0.5 \mathrm{~s}$. The TEM foils $(5 \mathrm{~mm} \times 2 \mathrm{~mm} \times 30 \mu \mathrm{m})$ [23] were thinned by twin-jet electropolishing in ethanol solution containing $5 \%$ perchloric acid at $-30{ }^{\circ} \mathrm{C}$.

\section{Results and discussion}

\subsection{ST and NT microstructure}

CG 304 SS has an austenite structure and could have various plastic deformation mechanisms at different strain rates $(\dot{\varepsilon})$ [24], resulting in different microstructures during SMAT processes. At relatively lower $\dot{\varepsilon}$ of $\sim 20-3 \times 10^{3} \mathrm{~s}^{-1}$, NCs can be obtained; at higher $\dot{\varepsilon}$ of $\sim 2 \times 10^{4}-1.2 \times 10^{5} \mathrm{~s}^{-1}$, deformation twins prevail [11, 24-27]. Martensitic phase transformation also occurs, depending on the deformation conditions. In Table 1, we summarize the measured microstructure information of all four types of gradient $304 \mathrm{SS}$, 
including phase, grain size, ST and/or NT, individual layer thickness, and volume fraction. Since ST and/or NT are the basis of all materials studied here, in Fig. 2 we present a detailed microscopic study of Type-II 304 SS that consists of STs and NTs. From the SEM image shown in Fig. 2a, the twin density is found to decrease into the depth direction. Under TEM, high-density NTs are observed near the very top surface, Fig. 2b. Since these twin boundaries (TBs) are formed via severe plastic deformation, defective nature of TBs is evident with copies dislocations tangled in between and at TBs. Kinked TBs are evident [28, 29], as better revealed by a high-resolution TEM image shown in Fig. 2c. The $\lambda$ is measured from $\sim 1.6-6.9 \mathrm{~nm}$. Careful examination of a large number of grains by tilting to $<011>$ orientation suggests that bundles of fine NTs $(\lambda<20$ $\mathrm{nm}$ ) exist in most grains. Underneath the NT layer (towards the right side of Fig. 2a), multiple intersection twins with a $\lambda$ of $\sim 50-400 \mathrm{~nm}$ are observed, Fig. 2 d. Statistical studies indicate that the average $\lambda$ increases from $\sim 80 \mathrm{~nm}$ at the very surface (Fig. $2 \mathrm{e}$ ) to $\sim 350 \mathrm{~nm}$ (Fig. 2f) in the center. Our quantitative measurements of twin density of Type-II $304 \mathrm{SS}$ indicate that it has a broad variation, from $\sim 70 \%$ on the surface to $\sim 40 \%$ in the center, Fig. 2g. This suggests that the as-fabricated Type-II material contains twin-density gradient as well.

\subsection{Tensile properties}

The engineering tensile stress-strain curves of all four types of gradient 304 SS are shown in Fig. 3a. For reference, the stress-strain curve of a CG 304 SS is also included. There are several salient features in these tensile data. First, we observe that all four types of materials show a good balance of strength and ductility, with Type-III sample exhibiting the highest yield strength of $966 \pm 8 \mathrm{MPa}$ and an engineering tensile elongation to failure $(\mathrm{TEF} \%)$ of $37 \pm 2 \%$, Table 2 . In this material, however, the addition of $\mathrm{NC}$ layers appears to comprise its useful tensile ductility as the observed TEF\% is substantially less than other three samples. Second and surprisingly, Type-II 304 SS, which has a relatively simple gradient of STs and NTs, shows a high yield strength of 
$820 \pm 9 \mathrm{MPa}(3 \mathrm{x}$ that of CG $304 \mathrm{SS}, 268 \pm 3 \mathrm{MPa}$ ) yet with a high engineering TEF\% of $65 \pm 3 \%$ that is comparable to CG $304 \mathrm{SS}(71 \pm 3 \%)$. This CG-type tensile ductility combined with high strength is quite impressive. Third, among four materials we observe a relatively "poor" combination of strength and ductility for Type-IV 304 SS. This is somewhat counterintuitive to the common belief that materials with a broad distribution of length scales will have an excellent set of mechanical properties. Instead our results here indicate that the gradient $\mathrm{ST}+\mathrm{NT}$ has the best performance. These results underscore the advantageous properties of TBs compared to grain boundaries (GBs) [30].

For structural materials, toughness is one of the most important parameters to evaluate their usefulness. The toughness of our steels can be quantitatively compared by integrating the stress-strain curves. It is equivalent to the energy of mechanical deformation per unit volume prior to fracture as

$$
\text { energy/volume }=\int_{0}^{\varepsilon_{f}} \sigma d \varepsilon
$$

where $\varepsilon, \varepsilon_{\mathrm{f}}$ and $\sigma$ are true strain, fracture strain and true stress, respectively. To better evaluate the toughness enhancement or reduction over CG materials, we normalize both the $\mathrm{TEF} \%$ and the toughness against respective CG $304 \mathrm{SS}$ values, the results of which are shown in Fig. 3b. With a normalized toughness value of 1.5, Type-II (ST+NT) 304 SS has emerged as a material with the best combination of strength and toughness. Type-I (ST) sample ranks the second with a normalized toughness value of 1.2. The other two materials (Type-III and Type-IV), both of which use NC layers as strengthening agent, show a slight degraded toughness compared to the CG 304 SS. Importantly, we observe that the toughness and ductility achieved in all of our gradient samples are substantially better than those of various high-strength 304 SS reported in the literature [12-19], including NC [15], UFG [14], UFG embodied with NTs [18], martensite-strengthened structure $[12,13,16]$, and the mixture of the NC, UFG and martensite $[17,19]$. These data underscore generally the advantageous properties of gradient materials. 
From tensile results, we hypothesize that the high-density STs and NTs in Type-II 304 SS make crucial contribution to the excellent combination of strength and toughness, which may be related to the unique work-hardening ability of twin structures. We calculate the normalized work-hardening rate $(\Theta)$ as

$$
\Theta=\frac{1}{\sigma}\left(\frac{\partial \sigma}{\partial \varepsilon}\right)_{\dot{\varepsilon}}
$$

The $\Theta$ values of four types of gradient steels are shown in Fig. 4. Type-IV 304 SS seems to exhibit a similar work hardening trend to CG $304 \mathrm{SS}$, both of which are signaled by an interesting work-hardening zone (pointed with arrows). The sudden increase of $\Theta$ observed inside this zone may be attributed to the martensitic transformation of CG grains [31]. In comparison, such work hardening zone does not present in three other samples. Type-I (ST) structure shows a quick decay of $\Theta$ as plastic strain progresses (Fig. 4b), a behavior that is different from other three materials. Type-III $(\mathrm{ST}+\mathrm{NT}+\mathrm{NC}) 304 \mathrm{SS}$ exhibits a lack of strain hardening capability, likely due to the existence of NC grains. Most intriguingly, we find that Type-II 304 SS exhibits an amazingly stable $\Theta$ value of 1.3 after yielding. According to the Consid re criterion, when $\Theta$ is larger than 1 , the onset of necking is delayed [32], leading to a large uniform tensile elongation [33]. Indeed, the engineering uniform elongation of Type-II 304 SS reaches as high as $53 \pm 3 \%$, Table 2. This persistent and stable value of $\Theta$ observed in Type-II steel is surprising and will be investigated in the next few sections.

\subsection{Postmortem microstructure of Type-II 304 SS}

To investigate the deformation mechanisms of ST+NT structures, we examined the postmortem microstructure of Type-II 304 SS after tensile fracture. The evolution of both ST and NT areas was investigated, as illustrated in Fig. 5a. In the NT zone after tensile deformation, we find two types of microstructures: martensitic phase $\left(\alpha^{\prime}\right)$ and very fine NTs, Fig. 5b. These NT bundles exhibit a narrow $\lambda$ distribution $(\sim 2-10 \mathrm{~nm})$. The lath-shape martensitic phase is also in the nanoscale region with a width of $\sim 30-100 \mathrm{~nm}$. It appears that the newly formed $\alpha^{\prime}$ martensitic phase preferentially nucleates at TBs and 
grows along the twin interfaces. The selected-area electron diffraction (SAED) in Fig. 5c indicates that the material is composed of $\alpha^{\prime}$ martensite, $\gamma$ austenite and twins with a well-known Kurdjumov-Sachs orientation relationship, $[\overline{1} 10]_{\gamma} / /[1 \overline{1} 0]_{t w i n} / /[1 \overline{1} 1]_{\alpha^{\prime}}$. In contrast, we observed mainly $\alpha^{\prime}$-martensitic phase in ST zone with very few twins left, Fig. 5d. The martensitic phase exhibits irregular shape with a lath width of $\sim 100-600 \mathrm{~nm}$ that is significantly wider than those seen in NT zone. These observations suggest that TBs can effectively regulate martensite nucleation and/or growth during the phase transformation. As a result, TBs could alter the $\Theta$ of gradient 304 SS seen in Fig. 4.

\subsection{In-situ TEM}

The excellent mechanical properties observed in Type-II (ST+NT) 304 SS (i.e., high strength, high ductility and high toughness) may open up new opportunities to defeat strength-toughness confliction in materials science $[2,21,34,35]$. It is thus important to understand the underlying deformation mechanisms in order to develop a general strategy to improve strength and ductility in $304 \mathrm{SS}$ and many other materials [36]. Our postmortem microstructure characterization in Fig. 5 provides some hints on the ongoing deformation mechanisms, including martensitic phase transformation, disappearance of STs and NTs, and dislocation activities. Twinning and detwinning could also be active but are difficult to judge from postmortem microstructure. The unusual strain hardening behavior seen in Fig. 4 for Type-II 304 SS suggests the important interplays among all above possible plastic deformation mechanisms. To further elucidate these rather complex deformation processes, we have performed in-situ TEM studies of this material.

Twinning and detwinning in NT structures We first examined the processes that result in $\alpha+\mathrm{NT}$ microstructure seen in Fig. 5b. Total seven in-situ TEM experiments (via both bright-field and dark-field imaging techniques) were carried out with samples prepared from NT zone. In all these experiments, we observed successive co-activations of twinning and detwinning, as shown in Fig. 6 (dark-field TEM). The red solid arrows and dashed light blue arrows in the figure point at twinning and detwinning processes, 
respectively. The TBs of interest are labeled with a number of 1-7. Two types of twinning processes were observed: the sudden appearance of a new TB (TB6, $c \rightarrow d$ and $g \rightarrow h$ ) and the growth of an existing twin plane (TB4, $\mathrm{a} \rightarrow \mathrm{b}$ and $\mathrm{e} \rightarrow \mathrm{f}$; TB7, $\mathrm{c} \rightarrow \mathrm{d}$ and $\mathrm{g} \rightarrow \mathrm{h}$ ). Conversely, fully detwinning (TB2, $\mathrm{a} \rightarrow \mathrm{b}$ and $\mathrm{e} \rightarrow \mathrm{f}$ ) and partially detwinning (TB3, $\mathrm{c} \rightarrow \mathrm{d}$ and $\mathrm{g} \rightarrow \mathrm{h}$ ) occurred. The migration of TB was also observed. For example, TB5 was recorded to migrate to the left by $6 \mathrm{~nm}$ from Fig. 6c to Fig. 6d. The extensively detwinning and TB migration observed in NT zone generally agree with recent experiments and molecular dynamics simulations that more defective TBs or TBs with smaller $\lambda$ are prone to detwinning $[28,37,38]$.

Martensitic transformation in NT zone In addition to twinning and detwinning phenomena, our in-situ TEM revealed mechanisms of martensitic transformation in NT zone. From six in-situ TEM experiments, we found that the martensitic transformation predominantly occurred in NTs with larger $\lambda$. An example of martensitic transformation, together with twinning and detwinning activities, is shown in Fig. 7. Three primary twin lamellar are labeled as "A, B, and C" in Fig.7a with $\lambda=60,100$, and $80 \mathrm{~nm}$, respectively. Numerous secondary TBs are observed inside domain "A and B" but not in "C". The SAED pattern shown in the inset of Fig. 7e confirms intersection twins. Upon an applied force, $\alpha$ ' martensitic phase transformation was observed to occur inside domain " $\mathrm{C}$ ", which was nucleated from TB between B and C, Fig. 7a-d and illustrations Fig. 7e-h. The SAED pattern in the inset of Fig. $7 f$ confirms the $\alpha$ martensitic phase formation. As the sample was continuously strained, we observed that the $\alpha$-martensite phase propagated along the TB in both directions, Fig. 7g-h. A dark-field TEM image of the $\alpha$ martensitic phase is displayed in the inset of Fig. 7h, together with its corresponding SAED pattern. In the meantime, twinning and detwinning activities were seen inside twin domain " $\mathrm{A}$ and B". For clarity, new formed TBs are labeled with red solid lines and detwinned TBs with dashed light blue lines. An interesting observation is that twinning and detwinning are much more active in domain " $\mathrm{A}$ " than in " $\mathrm{B}$ " because of its high twin-density. 
Implications to work hardening behavior From the above in-situ TEM results, we conclude that (1) twinning and detwinning are dominant events in NTs with $\lambda \sim 2-5 \mathrm{~nm}$, and (2) martensitic transformation occurs inside NT domain with $\lambda$ becomes larger. The martensitic transformation is known to increase the $\Theta$ of 304 SS, as body-centered cubic (BCC) $\alpha$ is much harder than face-centered cubic (FCC) $\gamma$ austenitic phase. However, as $\gamma$ phase depletes and $\alpha$ volume percentage increases, the driving force for martensitic phase nucleation decreases, leading to a decreased $\Theta$. Fast martensitic phase transformation could eventually make 304 SS less ductile due to the quick depletion of strain hardening capability. The mechanisms that help to slow down the martensitic transformation such as those we observed in NTs are beneficial to sustain a high $\Theta$. In Fig. 4, we observe that ST (Type-I) microstructure has a fast decay of $\Theta$ after yielding, consistent with the large $\alpha$ lath width observed inside ST structures. In contrast, a sustained $\Theta=1.3$ is observed for Type-II 304 SS, suggesting that the slow martensitic transformation due to the confinement of $\alpha$ in between TBs of NT zones could play a beneficial role. However, the loss of ductile FCC $\gamma$ austenite phase due to phase transformation in Type-II 304 SS needs to be compensated by other strain hardening mechanisms in order to maintain the constant $\Theta$. This would suggest additional twinning and dislocation-twin interactions, as indeed have been observed in our in-situ TEM studies. Earlier simulations further suggest that materials with gradient microstructure or under gradient stresses will have additional work hardening ability via geometrically necessary dislocations and complex stress states inside materials [5, 39-41]. These work hardening mechanisms are not easily available in uniform microstructure materials but are expected to be effective in our ST+NT microstructure. The gradient twin density seen in Type-II 304 SS (Fig. 2g) is likely to offer additional strain hardening capacity by accumulating dislocations inside grains with low density twins - a mechanism that has been reported in NT-Ag [10].

The detwinning mechanisms seen in Type-II 304 SS are not surprising, considering 
the small $\lambda$ and defective nature of these TBs. However, we did not observe the overall reduction of twin-density in our in-situ TEM experiments even with the ultrathin sample geometries (Supplementary Movie 1 and Movie 2), suggesting that the detwinning phenomenon we saw under in-situ TEM is a reactive mechanism to different stress states and may not necessarily cause softening [42-44]. We remain cautious, however, that the net effect of twinning, detwinning, and martensitic phase transformation on strain hardening behavior requires more in-depth theoretical investigation.

\subsection{Fracture behavior}

To gain further insights on the deformation mechanisms of gradient $304 \mathrm{SS}$, we examined and compared the fracture surfaces of four types of samples after tensile failure, Fig. 8. Perhaps not surprisingly, both Type-III and Type-IV 304 SS exhibit gradient dimple patterns, with the NC layer showing brittle fracture surface, Fig. 8c, d. This suggests nonhomogeneous deformation nature of these two samples before fracture. In comparison, Type-I (ST) 304 SS reveals relatively uniform dimple patterns (Fig. 8a), although large voids or crack-like features (pointed with arrows) are visible. These microscopic cracks and voids are likely caused by the interfaces of austenite and martensite, which are the known source of crack initiation in 304 SS. Strikingly, despite its gradient microstructure, Type-II 304 SS shows rather uniform and fine dimple patterns. Occasionally, large voids are observed inside ST layer but with much less frequencies. The fracture features in Fig. 8b suggest that Type-II 304 SS deforms relatively uniformly before it raptures. This behavior is quite different from Type-III and Type-IV gradient samples, where the failure seems to originate from NC layer with little plasticity, Fig. 8c, d. In contrast, NT layers in Type-II 304 SS are capable of offering additional strain hardening mechanisms under high stresses $[45,46]$, help to stabilize tensile deformation and lead to uniform and fine fracture dimples [47].

\subsection{Retaining high strength and high toughness via ST and NT gradient structures}

Our experimental results above have established that $\mathrm{ST}+\mathrm{NT}$ gradient microstructure 
is a unique design strategy that allows for high-strength and has toughness that is $\sim 50 \%$ better than the CG counterpart. Our findings contrast with the popular belief that $\mathrm{NC}+\mathrm{UFG}+\mathrm{CG}$ (i.e., grain-size-based gradient design) gradient materials can outperform CG structures. The results above indicate that some gradient materials (for example, Type-III and Type-IV 304 SS) cannot surpass the toughness of CG counterparts, making them less attractive for practical applications despite often reported high-strength.

One core design of our strategy is to use ST structure as the main load carrier instead of commonly used CG grains. This helps to substantially elevate the overall strength of the material without much sacrifice on tensile ductility. Another key design of our gradient microstructure is to use NT out layers to replace NC layers that have been popularly used in the literature $[4,5,17,18]$. Our approach has at least two advantages. First, unlike NC grains, which have little resistant to tensile strain and plasticity, NT structure is well known to be able to accommodate very large tensile strains. This can be easily seen in the rather uniform dimple patterns on the fracture surface of Type-II 304 SS, Fig. 8b. Second, as the tensile deformation is sensitive to surface flaws and easy fracture [48], NTs are a better choice as they have higher resistant to cracking than NC grains [49, 50]. As our approach does not require an ultrathick layer of NT structures, it is likely that our ST+NT strategy is applicable to other metals and alloys, including those materials with high stacking fault energies. Recent experiments have indeed demonstrated the feasibility of fabricating thin high-density NT structure even in Al [51, 52]. These design advantages have clearly made our approach attractive for engineering applications.

\section{Concluding remarks}

Using ST structure as the baseline design strategy, we have investigated several gradient microstructures in $304 \mathrm{SS}$. We find that $\mathrm{ST}+\mathrm{NT}$ gradient materials outperform other hierarchical structures that contain NC and CG grains. The main conclusions of our work can be summarized as follows:

(1) High-strength and high ductility are observed in all gradient structures investigated in 
this study, including ST, ST+NT, ST+NT+NC, and ST+NC+UFG+CG.

(2) Both ST and ST+NT gradient materials have toughness that is substantially higher than that of the CG counterparts. In contrast, the toughness of $\mathrm{ST}+\mathrm{NT}+\mathrm{NC}$ and $\mathrm{ST}+\mathrm{NC}+\mathrm{UFG}+\mathrm{CG}$ is at par with that of the $\mathrm{CG}$ materials. The work hardening rate of $\mathrm{ST}+\mathrm{NT}$ is found to have a stable value of 1.3 , beneficial for delaying necking and maintaining high ductility.

(3) STs and NTs in $304 \mathrm{SS}$ are found to suppress the martensitic phase transformation through the confinement of the new phase within the twin lamellar.

(4) Twinning and detwinning are found very active in NT region of 304 SS. Detwinning is observed to happen mostly in twin regions with $\lambda \sim 2-5 \mathrm{~nm}$, whereas twinning occurs through the sudden appearance of a new TB or the growth of an existing twin plane.

(5) Type-II (ST+NT) 304 SS exhibits uniform fracture dimple patterns, in sharp contrast with the gradient dimple patterns seen in Type-III and Type-IV 304 SS, both of which has NC outside layers as strengthening agents.

(6) The design strategy of ST+NT gradient material has shown promising advantages over grain-size-based gradient materials. Our approach is likely applicable to other metals and alloys.

\section{Acknowledgement}

The authors acknowledge the support from NSFC (Nos. 11202134, 51271123, 11202183 and 11572281). L.J. gratefully acknowledges financial support provided by the MOST through the National Basic Research Program of China (973 Program) under the grant 2012CB932203. The work at Lawrence Livermore National Laboratory was supported by the U.S. Department of Energy under Contract DE-AC52-07NA27344.

\section{References}

[1] K. Lu. Making strong nanomaterials ductile with gradients, Science 345 (2014) 1455-1456.

[2] Y.M. Wang, M.W. Chen, F.H. Zhou, E. Ma. High tensile ductility in a nanostructured metal, Nature 419 (2002) 912-915.

[3] X.L. Wu, F. Yuan, M. Yang, P. Jiang, C. Zhang, L. Chen, Y. Wei, E. Ma. Nanodomained 
nickel unite nanocrystal strength with coarse-grain ductility, Sci. Rep. 5 (2015) 11728.

[4] T.H. Fang, W.L. Li, N.R. Tao, K. Lu. Revealing Extraordinary Intrinsic Tensile Plasticity in Gradient Nano-Grained Copper, Science 331 (2011) 1587-1590.

[5] X. Wu, P. Jiang, L. Chen, F. Yuan, Y.T. Zhu. Extraordinary strain hardening by gradient structure, Proc. Natl. Acad. Sci. U. S. A. 111 (2014) 7197-7201.

[6] Y. Wei, Y. Li, L. Zhu, Y. Liu, X. Lei, G. Wang, Y. Wu, Z. Mi, J. Liu, H. Wang, H. Gao. Evading the strength- ductility trade-off dilemma in steel through gradient hierarchical nanotwins, Nat. Commun. 5 (2014) 3580.

[7] L. Lu, Y.F. Shen, X.H. Chen, L.H. Qian, K. Lu. Ultrahigh strength and high electrical conductivity in copper, Science 304 (2004) 422-426.

[8] E. Ma, Y.M. Wang, Q.H. Lu, M.L. Sui, L. Lu, K. Lu. Strain hardening and large tensile elongation in ultrahigh-strength nano-twinned copper, Appl. Phys. Lett. 85 (2004) 4932-4934.

[9] I.J. Beyerlein, X. Zhang, A. Misra. Growth Twins and Deformation Twins in Metals, Annu. Rev. Mater. Res. 44 (2014) 329-363.

[10] R.T. Ott, J. Geng, M.F. Besser, M.J. Kramer, Y.M. Wang, E.S. Park, R. LeSar, A.H. King. Optimization of strength and ductility in nanotwinned ultra-fine grained Ag: Twin density and grain orientations, Acta Mater. 96 (2015) 378-389.

[11]A.Y. Chen, H.H. Ruan, J. Wang, H.L. Chan, Q. Wang, Q. Li, J. Lu. The influence of strain rate on the microstructure transition of 304 stainless steel, Acta Mater. 59 (2011) 3697-3709.

[12] B.R. Kumar, S. Sharma, B. Mahato. Formation of ultrafine grained microstructure in the austenitic stainless steel and its impact on tensile properties, Mater. Sci. Eng. A 528 (2011) 2209-2216.

[13] M. Milad, V. Zreiba, F. Elhalouani, C. Baradai. The effect of cold work on structure and properties of AISI 304 stainless steel, J. Mater. Process. Technol. 203 (2008) 80-85.

[14] S. Qu, C.X. Huang, Y.L. Gao, G. Yang, S.D. Wu, Q.S. Zang, Z.F. Zhang. Tensile and compressive properties of AISI 304L stainless steel subjected to equal channel angular pressing, Mater. Sci. Eng. A 475 (2008) 207-216.

[15] I. Shakhova, V. Dudko, A. Belyakov, K. Tsuzaki, R. Kaibyshev. Effect of large strain cold rolling and subsequent annealing on microstructure and mechanical properties of an austenitic stainless steel, Mater. Sci. Eng. A 545 (2012) 176-186.

[16] Y.F. Shen, N. Jia, Y.D. Wang, X. Sun, L. Zuo, D. Raabe. Suppression of twinning and phase transformation in an ultrafine grained $2 \mathrm{GPa}$ strong metastable austenitic steel: Experiment and simulation, Acta Mater. 97 (2015) 305-315.

[17] C. Ye, A. Telang, A.S. Gill, S. Suslov, Y. Idell, K. Zweiacker, J.M.K. Wiezorek, Z. Zhou, D. Qian, S.R. Mannava, V.K. Vasudevan. Gradient nanostructure and residual stresses induced by Ultrasonic Nano-crystal Surface Modification in 304 austenitic stainless steel for high strength and high ductility, Mater. Sci. Eng. A 613 (2014) 274-288.

[18] C.X. Huang, W.P. Hu, Q.Y. Wang, C. Wang, G. Yang, Y.T. Zhu. An ideal ultrafine-grained structure for high strength and high ductility, Mater. Res. Lett. 3 (2015) 88-94.

[19] A. Momeni, S.M. Abbasi. Repetitive Thermomechanical Processing towards Ultra Fine Grain Structure in 301, 304 and 304L Stainless Steels, J. Mater. Sci. Tech. 27 (2011) 338-343. 
[20] H.Y. Yi, F.K. Yang, N.R. Tao, K. Lu. Comparison of strengh-ductility combinations between nanotwinned austenite and martensite-austenite stainless steels, Mater. Sci. Eng. A 647 (2015) 152-156.

[21] R.O. Ritchie. The conflicts between strength and toughness, Nature Mater. 10 (2011) 817-822.

[22] H.W. Huang, Z.B. Wang, J. Lu, K. Lu. Fatigue behaviors of AISI 316L stainless steel with a gradient nanostructured surface layer, Acta Mater. 87 (2015) 150-160.

[23] H. Wang, A. Nie, J. Liu, P. Wang, W. Yang, B. Chen, H. Liu, M. Fu. In situ TEM study on crack propagation in nanoscale Au thin films, Scr. Mater. 65 (2011) 377-379.

[24] Z. Yanushkevich, A. Belyakov, R. Kaibyshev. Microstructural evolution of a 304-type austenitic stainless steel during rolling at temperatures of 773-1273 K, Acta Mater. 82 (2015) 244-254.

[25] A.Y. Chen, H.H. Ruan, J.B. Zhang, X.R. Liu, J. Lu. Introducing a hierarchical structure for fabrication of a high performance steel, Mater. Chem. Phys. 129 (2011) 1096-1103.

[26] H.W. Zhang, Z.K. Hei, G. Liu, J. Lu, K. Lu. Formation of nanostructured surface layer on AISI 304 stainless steel by means of surface mechanical attrition treatment, Acta Mater. 51 (2003) 1871-1881.

[27] S. Mahajan. Critique of mechanisms of formation of deformation, annealing and growth twins: Face-centered cubic metals and alloys, Scr. Mater. 68 (2013) 95-99.

[28] Y.M. Wang, F. Sansoz, T. LaGrange, R.T. Ott, J. Marian, T.W. Barbee, Jr., A.V. Hamza. Defective twin boundaries in nanotwinned metals, Nature Mater. 12 (2013) 697-702.

[29] N. Lu, K. Du, L. Lu, H.Q. Ye. Transition of dislocation nucleation induced by local stress concentration in nanotwinned copper, Nat. Commun. 6 (2015) 7648.

[30] K. Lu, L. Lu, S. Suresh. Strengthening Materials by Engineering Coherent Internal Boundaries at the Nanoscale, Science 324 (2009) 349-352.

[31] N. Li, Y.D. Wang, W.J. Liu, Z.N. An, J.P. Liu, R. Su, J. Li, P.K. Liaw. In situ X-ray microdiffraction study of deformation-induced phase transformation in 304 austenitic stainless steel, Acta Mater. 64 (2014) 12-23.

[32] E.W. Hart. Theory of tensile test, Acta Metall. 15 (1967) 351-355.

[33] Y.M. Wang, E. Ma. Three strategies to achieve uniform tensile deformation in a nanostructured metal, Acta Mater. 52 (2004) 1699-1709.

[34] Y.T. Zhu, X.Z. Liao. Nanostructured metals - Retaining ductility, Nature Mater. 3 (2004) 351-352.

[35] R. Valiev. Materials science - Nanomaterial advantage, Nature 419 (2002) 887-887.

[36] E. Ma. Eight routes to improve the tensile ductility of bulk nanostructured metals and alloys, Jom 58 (2006) 49-53.

[37] J. Wang, N. Li, O. Anderoglu, X. Zhang, A. Misra, J.Y. Huang, J.P. Hirth. Detwinning mechanisms for growth twins in face-centered cubic metals, Acta Mater. 58 (2010) 2262-2270.

[38] L. Xu, D. Xu, K.N. Tu, Y. Cai, N. Wang, P. Dixit, J.H.L. Pang, J.M. Miao. Structure and migration of (112) step on (111) twin boundaries in nanocrystalline copper, J. Appl. Phys. 104 
(2008) 113717.

[39] S.S. Chakravarthy, W.A. Curtin. Stress-gradient plasticity, Proc. Natl. Acad. Sci. U. S. A. 108 (2011) 15716-15720.

[40] A. Arsenlis, D.M. Parks. Crystallographic aspects of geometrically-necessary and statistically-stored dislocation density, Acta Mater. 47 (1999) 1597-1611.

[41] D. Raabe, Z. Zhao, S.J. Park, F. Roters. Theory of orientation gradients in plastically strained crystals, Acta Mater. 50 (2002) 421-440.

[42] Y. Liu, J. Jian, Y. Chen, H. Wang, X. Zhang. Plasticity and ultra-low stress induced twin boundary migration in nanotwinned $\mathrm{Cu}$ by in situ nanoindentation studies, Appl. Phys. Lett. 104 (2014) 231910.

[43] A.M. Hodge, T.A. Furnish, C.J. Shute, Y. Liao, X. Huang, C.S. Hong, Y.T. Zhu, T.W. Barbee, Jr., J.R. Weertman. Twin stability in highly nanotwinned $\mathrm{Cu}$ under compression, torsion and tension, Scr. Mater. 66 (2012) 872-877.

[44] C.J. Shute, B.D. Myers, S. Xie, S.Y. Li, T.W. Barbee, Jr., A.M. Hodge, J.R. Weertman. Detwinning, damage and crack initiation during cyclic loading of $\mathrm{Cu}$ samples containing aligned nanotwins, Acta Mater. 59 (2011) 4569-4577.

[45] S. Mahajan, G.Y. Chin. Interaction of twins with existing substructure and twins in cobalt-iron alloys, Acta Metall. 22 (1974) 1113-1119.

[46] X. Zhang, A. Misra, H. Wang, M. Nastasi, J.D. Embury, T.E. Mitchell, R.G. Hoagland, J.P. Hirth. Nanoscale-twinning-induced strengthening in austenitic stainless steel thin films, Appl. Phys. Lett. 84 (2004) 1096-1098.

[47] Y.M. Wang, E. Ma, M.W. Chen. Enhanced tensile ductility and toughness in nanostructured Cu, Appl. Phys. Lett. 80 (2002) 2395-2397.

[48] Y.M. Wang, R.T. Ott, T. van Buuren, T.M. Willey, M.M. Biener, A.V. Hamza. Controlling factors in tensile deformation of nanocrystalline cobalt and nickel, Phys. Rev. B 85 (2012) 014101.

[49] Z. Zeng, X.Y. Li, L. Lu, T. Zhu. Fracture in a thin film of nanotwinned copper, Acta Mater. 98 (2015) 313-317.

[50] D. Farkas, S. Van Petegem, P.M. Derlet, H. Van Swygenhoven. Dislocation activity and nano-void formation near crack tips in nanocrystalline Ni, Acta Mater. 53 (2005) 3115-3123.

[51] D. Bufford, Y. Liu, J. Wang, H. Wang, X. Zhang. In situ nanoindentation study on plasticity and work hardening in aluminium with incoherent twin boundaries, Nat. Commun. 5 (2014) 4864.

[52] S. Xue, Z. Fan, Y. Chen, J. Li, H. Wang, X. Zhang. The formation mechanisms of growth twins in polycrystalline Al with high stacking fault energy, Acta Mater. 101 (2015) 62-70. 
Table 1. Microstructure characteristics of four types of gradient 304 SS. Symbols in the table: $\alpha^{\prime}$ : martensitic phase; $\gamma$ : austenite phase; $d$ : grain size; $\lambda$ : average twin spacing; $\mathrm{ST}=$ submicrotwins; $\quad \mathrm{NT}=$ nanotwins $; \quad \mathrm{NC}=$ nanocystalline $; \quad \mathrm{UFG}=$ ultrafine-grain; $\mathrm{CG}=$ coarse-grain.

\begin{tabular}{|c|c|c|c|c|c|c|}
\hline Type & Layer & Microstructure & $d(\lambda)$ & Volume fraction $(\%)$ & Layer depth $(\mu \mathrm{m})$ & Thickness (mm) \\
\hline \multirow{3}{*}{$\mathbf{I}$} & \multirow{3}{*}{ ST } & $\alpha^{\prime}$ & / & 6 & \multirow{3}{*}{400} & \multirow{3}{*}{$\sim 0.4$} \\
\hline & & $\gamma$ & $15 \mu \mathrm{m}$ & 94 & & \\
\hline & & twin & $0.1-1.2 \mu \mathrm{m}$ & 40 & & \\
\hline \multirow{6}{*}{ II } & \multirow{3}{*}{ ST } & $\alpha^{\prime}$ & l & 6 & \multirow{3}{*}{400} & \multirow{6}{*}{$\sim 0.7$} \\
\hline & & $\gamma$ & $15 \mu \mathrm{m}$ & 94 & & \\
\hline & & twin & $0.1-1.2 \mu \mathrm{m}$ & 40 & & \\
\hline & \multirow{3}{*}{ NT } & $\alpha^{\prime}$ & I & 6 & \multirow{3}{*}{150} & \\
\hline & & $\gamma$ & $1-15 \mu \mathrm{m}$ & 94 & & \\
\hline & & twin & $2-400 \mathrm{~nm}$ & $70-40$ & & \\
\hline \multirow{8}{*}{ III } & \multirow{3}{*}{$\mathrm{ST}$} & $\alpha^{\prime}$ & / & 6 & \multirow{3}{*}{400} & \multirow{8}{*}{$\sim 0.9$} \\
\hline & & $\gamma$ & $15 \mu \mathrm{m}$ & 94 & & \\
\hline & & twin & $0.1-1.2 \mu \mathrm{m}$ & 40 & & \\
\hline & \multirow{3}{*}{ NT } & $\alpha^{\prime}$ & / & 6 & \multirow{3}{*}{250} & \\
\hline & & $\gamma$ & $1-15 \mu \mathrm{m}$ & 94 & & \\
\hline & & twin & $2-400 \mathrm{~nm}$ & $70-40$ & & \\
\hline & \multirow{2}{*}{$\mathrm{NC}$} & $\alpha^{\prime}$ & 120 & 23 & \multirow{2}{*}{20} & \\
\hline & & $\gamma$ & 250 & 77 & & \\
\hline \multirow{7}{*}{ IV } & \multirow{2}{*}{ ST } & $\gamma$ & $1-15 \mu \mathrm{m}$ & 94 & \multirow{2}{*}{100} & \multirow{7}{*}{$\sim 1$} \\
\hline & & Twin & $500 \mathrm{~nm}$ & l & & \\
\hline & \multirow{2}{*}{$\mathrm{NC}$} & $\alpha^{\prime}$ & $10 \mathrm{~nm}$ & 95 & \multirow{2}{*}{20} & \\
\hline & & $\gamma$ & I & 5 & & \\
\hline & \multirow{2}{*}{ UFG } & $\alpha^{\prime}$ & $60 \mathrm{~nm}$ & 1 & \multirow{2}{*}{200} & \\
\hline & & $\gamma$ & $200 \mathrm{~nm}$ & 1 & & \\
\hline & $\mathrm{CG}$ & $\gamma$ & $15 \mu \mathrm{m}$ & 94 & 350 & \\
\hline
\end{tabular}


Table 2. A summary of tensile properties of gradient 304 SS. All the data shown are engineering values.

\begin{tabular}{cccccc}
\hline Type & Microstructure & $\begin{array}{c}\text { Yield strength } \\
(\mathrm{MPa})\end{array}$ & $\begin{array}{c}\text { Ultimate strength } \\
(\mathrm{MPa})\end{array}$ & $\begin{array}{c}\text { Elongation to } \\
\text { fracture }(\%)\end{array}$ & $\begin{array}{c}\text { Uniform elongation } \\
(\%)\end{array}$ \\
\hline I & ST & $582 \pm 9$ & $864 \pm 12$ & $62 \pm 4$ & $49 \pm 3$ \\
II & ST+NT & $820 \pm 9$ & $1012 \pm 13$ & $65 \pm 3$ & $53 \pm 3$ \\
III & ST+NT+NC & $966 \pm 8$ & $1069 \pm 9$ & $37 \pm 2$ & $30 \pm 2$ \\
IV & ST+ NC+UFG+CG & $610 \pm 5$ & $858 \pm 5$ & $50 \pm 2$ & $45 \pm 2$ \\
$304 \mathrm{SS}$ & $\mathrm{CG}$ & $268 \pm 3$ & $671 \pm 7$ & $71 \pm 2$ & $63 \pm 2$ \\
\hline
\end{tabular}



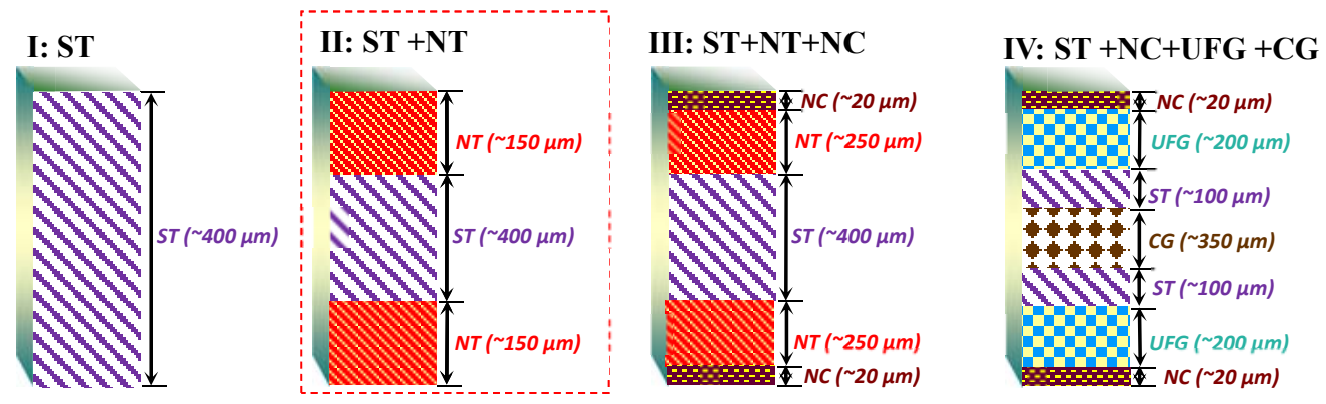

Figure 1. Schematic illustrations of four types of gradient 304 stainless steels (304 SS). Type-I 304 SS is composed of submicrometer twins (ST) with an average twin spacing $(100 \mathrm{~nm}<\lambda<1000 \mathrm{~nm})$. Type-II 304 SS contains ST-layer core ( $\sim 57$ vol.\%), sandwiched by two outside nanotwinned (NT, $\lambda<100 \mathrm{~nm}$ ) layers ( 43 vol.\%). Type-III $304 \mathrm{SS}$ $(\mathrm{ST}+\mathrm{NT}+\mathrm{NC})$ is formed by adding two more outside nanocrystalline $(\mathrm{NC})$ layers (grain size $d<100 \mathrm{~nm}$ ) on Type-II material. Type-IV $304 \mathrm{SS}$ includes four length scales, including NC, ultrafine-grains (UFG) $(100 \mathrm{~nm}<d<1000 \mathrm{~nm})$, ST and coarse-grains (CG, $\mathrm{d}>1 \mu \mathrm{m})$. 


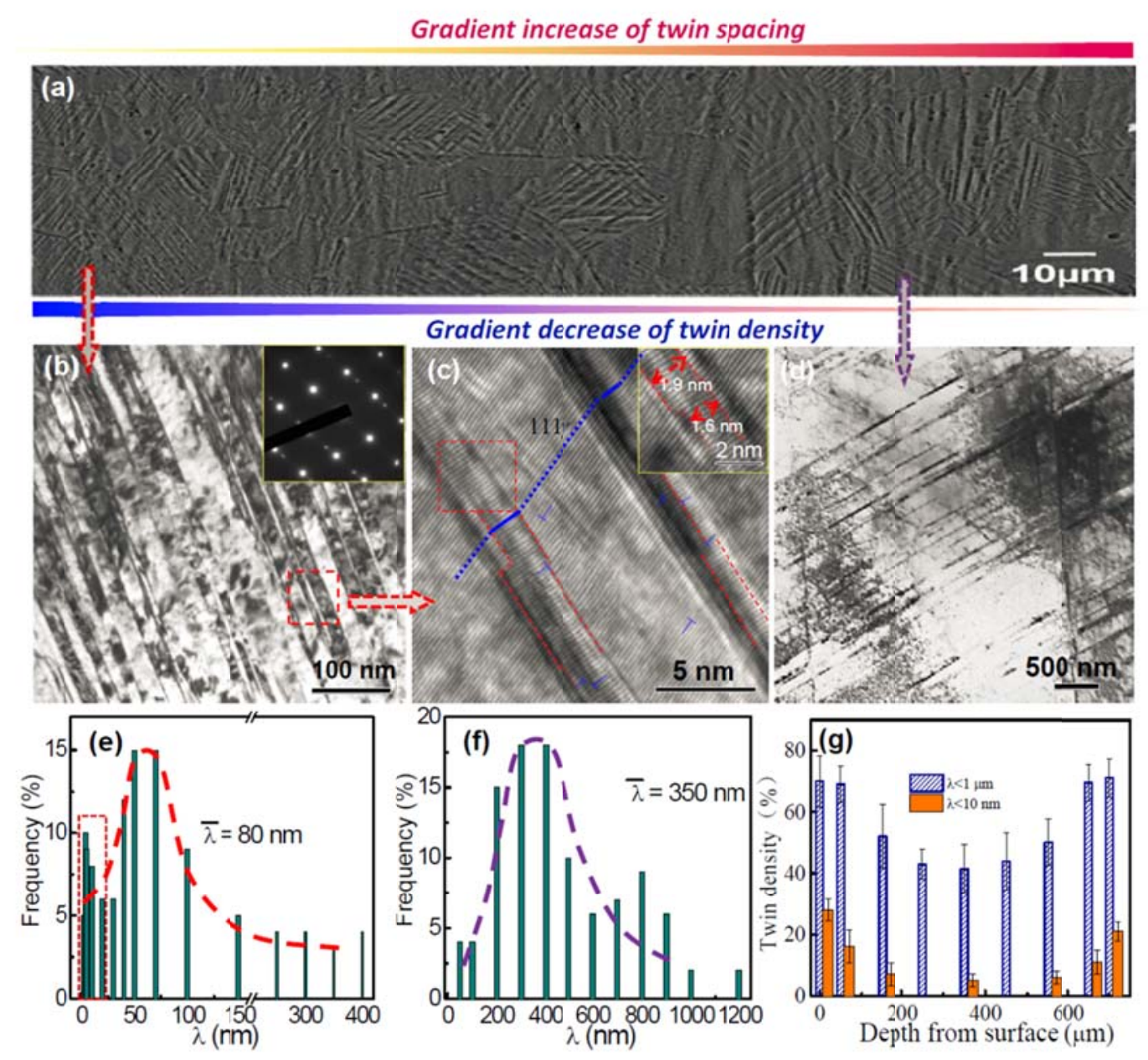

Figure 2. Submicrometer twin (ST) and nanotwin (NT) structures observed in Type-II 304 SS. (a) Cross-sectional SEM image from the top surface (left) to the center (right). (b) High-magnification TEM image of NTs on the top surface and its corresponding SAED pattern. Some dislocations are marked with a "T". (c) High-resolution TEM image taken from the $<110>$ zone axis marked in (b); (d) Bright-field TEM image of intersection STs located at $\sim 400 \mu \mathrm{m}$ depth. (e) and (f) Statistic distribution of the average twin spacing $(\lambda)$ corresponding to (b) and (d), respectively. (g) Twin-density $(\lambda<1 \mu \mathrm{m})$ distribution plot along the depth direction. 

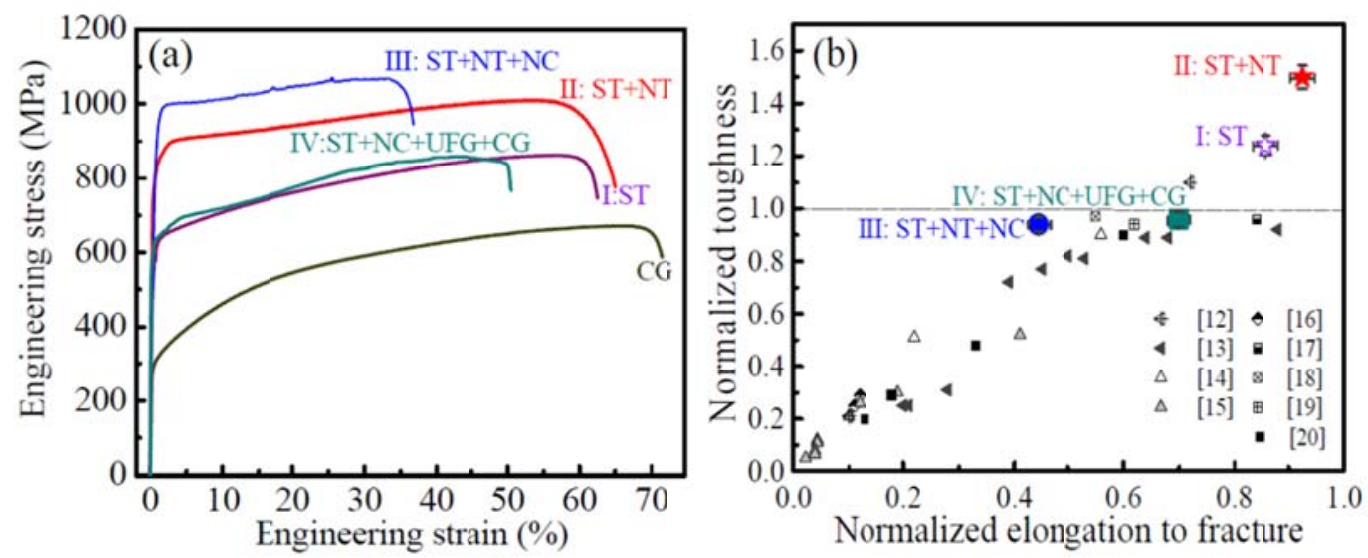

Figure 3. Tensile properties and toughness of various gradient 304 SS. (a) Tensile engineering stress-strain curves. (b) Normalized toughness of all four types of gradient 304 SS samples, together with other 304 SS data from the literature. See the main text for the definition of the normalized toughness and normalized elongation to failure. 

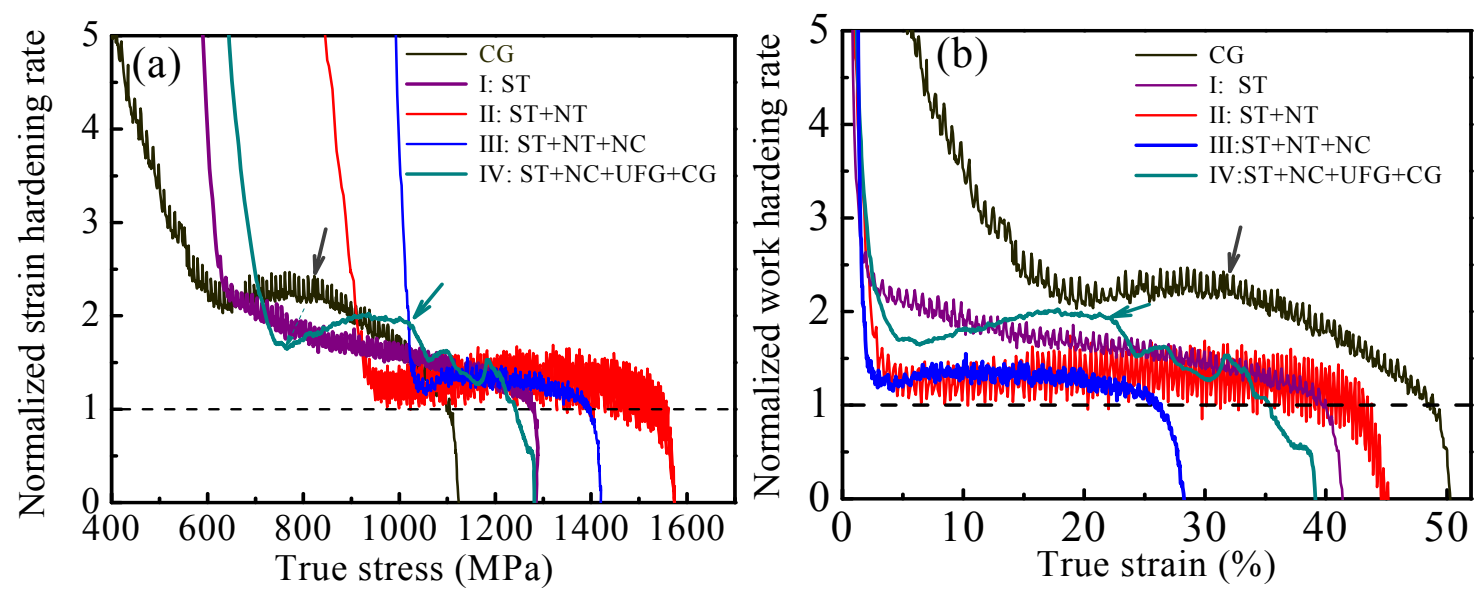

Figure 4. The work hardening behavior of four gradient $304 \mathrm{SS}$, in comparison with the CG data. The work hardening rate $(\Theta)$ is plotted against (a) true stress, and (b) true strain. 

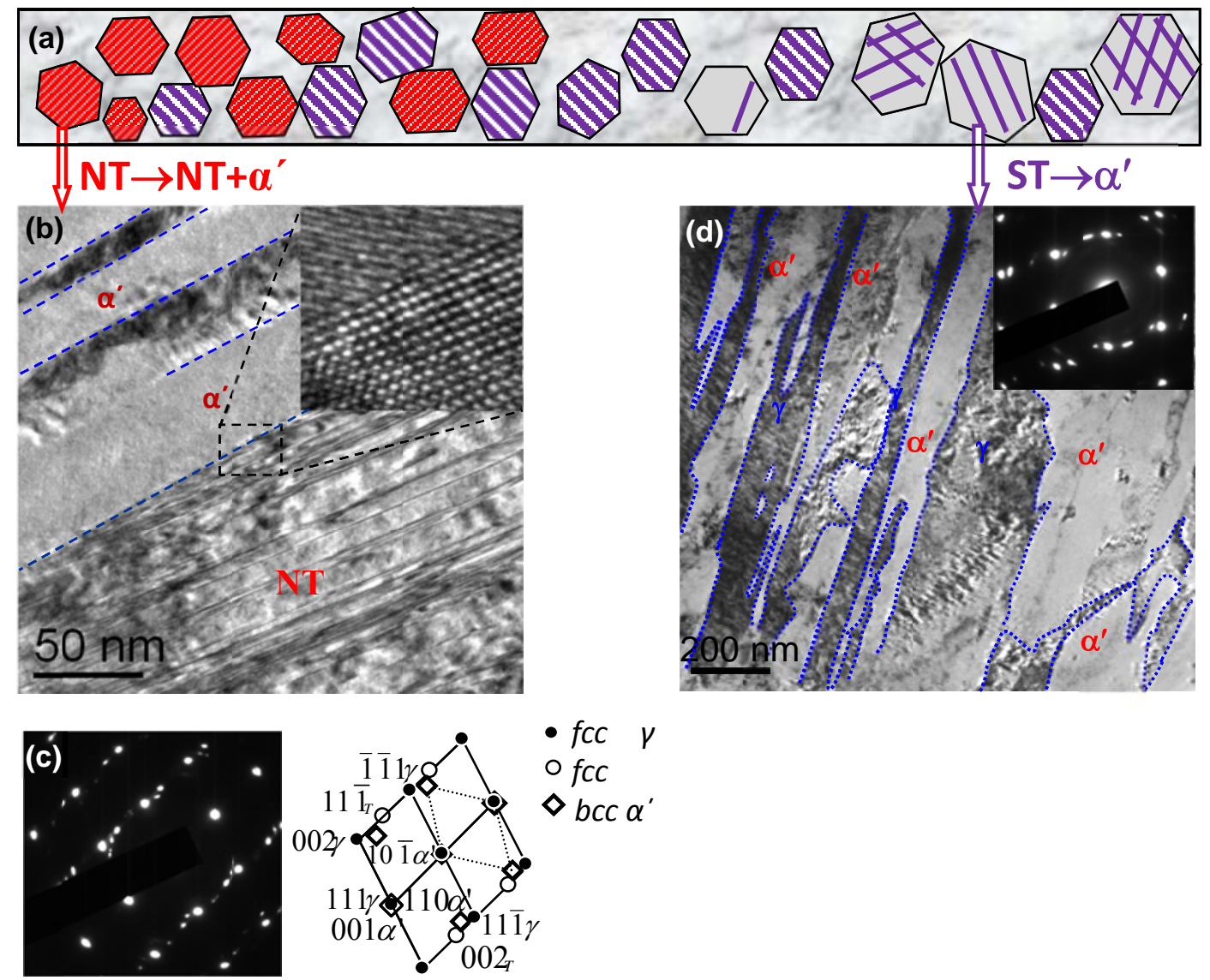

Figure 5. The postmortem microstructure of Type-II $304 \mathrm{SS}(\mathrm{ST}+\mathrm{NT})$ after tensile failure. (a) A schematic of the cross-sectional microstructure. (b) A bright-field TEM image taken from the NT zone, showing $\alpha^{\prime}$ martensite and nanotwins (NTs). (c) The SAED of (b), indexed pattern with axis of $[\overline{1} 10]_{\gamma} / /[1 \overline{1} 0]_{t w i n} / /[1 \overline{1} 1]_{\alpha^{\prime}}$. (d) A bright-field TEM image taken from the ST zone, showing lath $\alpha^{\prime}$ martensite phase. The inset in (b) shows the interface of austenite and martensite phases, and that in (d) is the corresponding SAED with the zone axis of $[\overline{1} 10]_{\gamma} / /[1 \overline{1} 1]_{\alpha^{\prime}}$. 


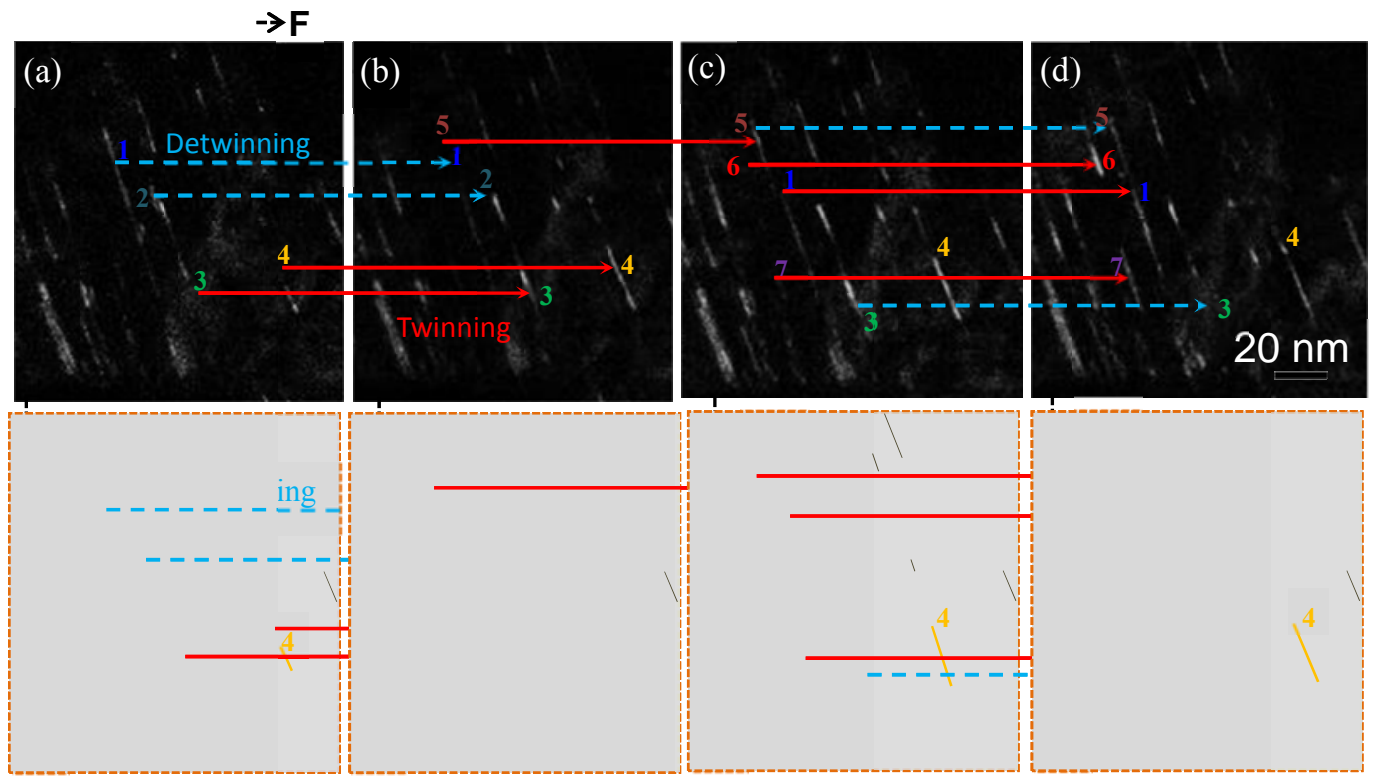

Figure 6. Sequential dark-field TEM images of nanotwin (NT) zone under tensile deformation. The timeframe from (a)-(d) is $0 \mathrm{~s}, 18 \mathrm{~s}, 30 \mathrm{~s}$, and $45 \mathrm{~s}$, respectively. Twinning and detwinning are observed to be active, which are indicated by red solid arrows and blue dashed arrows, respectively. The illustrations in (e-h) correspond to the top TEM images (a-d). Here the newly formed NTs are indicated by red solid lines, and the disappearance of NTs (i.e., detwinning) is represented by the light blue dash lines. The NTs, "1 and 2", occurs partially and totally detwinning, respectively, and NTs, "3 and 4", show partial twinning, as shown in (b) and (f). Accompanied with the detwinning of " 1 ", new NT is formed, indicated by " 5 " in (c). The frames in (g) and (h) show the slip of NTs. 


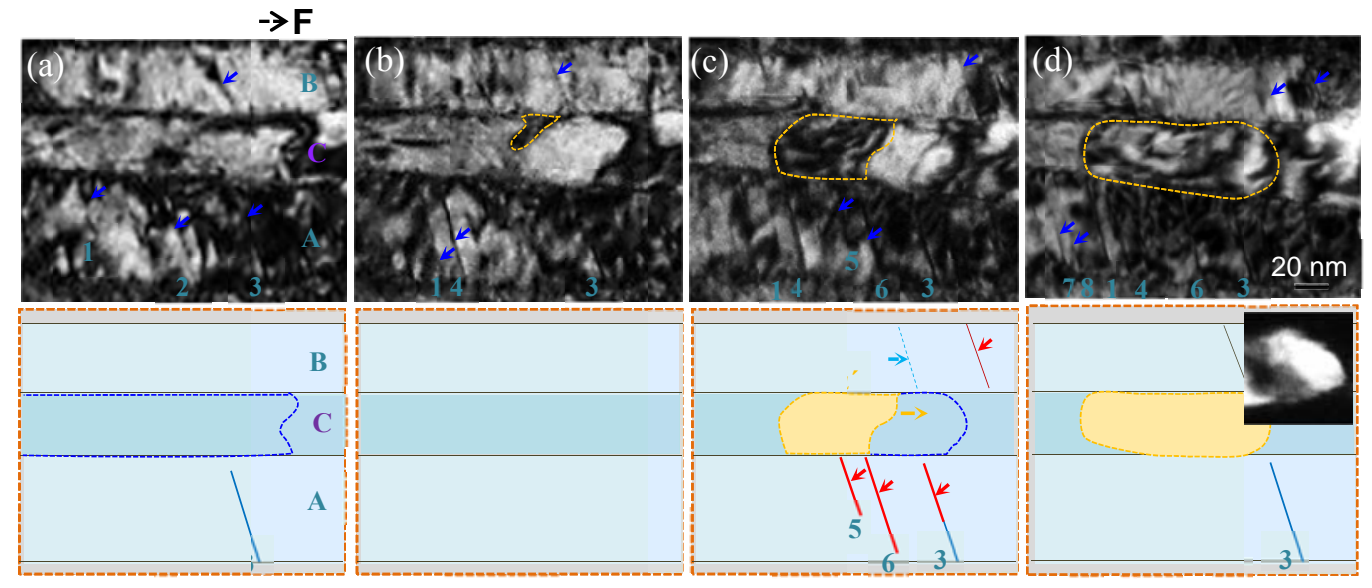

Figure 7. Sequential TEM images of $\alpha$ martensitic transformation in nanotwin (NT) zone under tensile stresses. The timeframe from (a)-(d) is $0 \mathrm{~s}, 11 \mathrm{~s}, 32 \mathrm{~s}$, and $54 \mathrm{~s}$, respectively. The illustrations in (e-h) correspond to the tope TEM images of (a-d). Three primary NT lamellar are labeled with a letter "A, B, and C". Secondary NTs can be seen inside twin lamellar "A and B". The intersection of multiple NTs is confirmed by the SAED pattern shown in the inset of (e). The nucleation and propagation $\alpha$ martensite phase inside "C" twin lamellar can be clearly seen in (b-d) and (f-h). The insets in (f) and (h) show the SAED patterns of the newly formed $\alpha$ martensite phase and its corresponding dark-field image, respectively. In the meantime, twinning and detwinning are observed inside "A and B" twin lamellar. Each twin-boundary (TB) is labeled with a number 1-8. For clarity, solid and dashed lines in (e-h) represent twinning and detwinning events, respectively. 

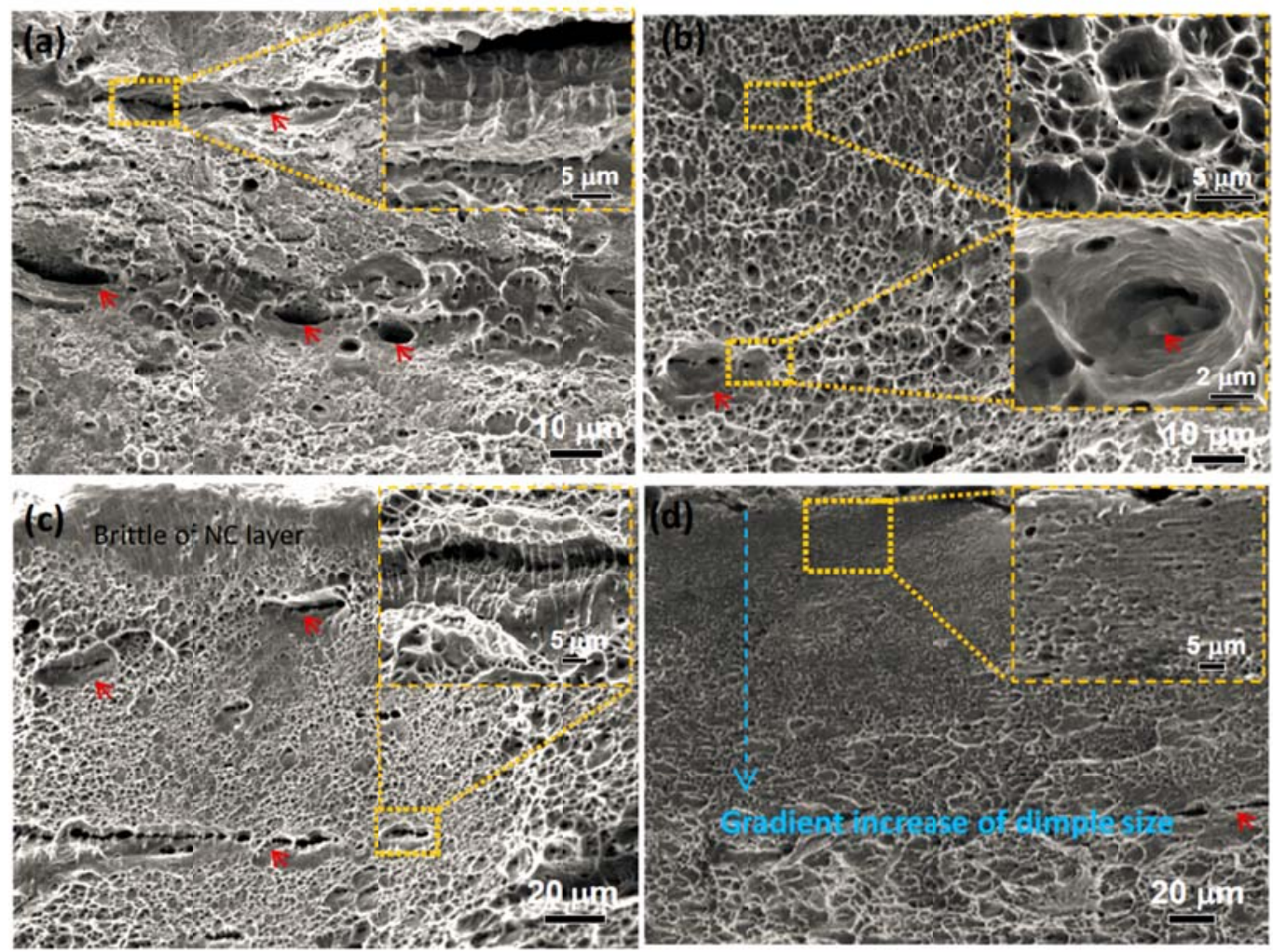

Figure 8. Fractographs of four types of gradient 304 SS. (a)-(d) Fracture surfaces of Type-I, Type-II, Type-III, and Type-IV 304 SS, respectively. Arrows in each figure point at large voids or microcracks. Note that Type-III and Type-IV show gradient dimple sizes, whereas Type-I and Type-II 304 SS have relatively uniform dimple distributions. Interestingly, Type-II $304 \mathrm{SS}$ has the smallest average dimple sizes. 